PROCEEDINGS OF THE

AMERICAN MATHEMATICAL SOCIETY

Volume 137, Number 11, November 2009, Pages 3709-3716

S 0002-9939(09)10023-0

Article electronically published on June 29, 2009

\title{
BICOMMUTANTS OF REDUCED UNBOUNDED OPERATOR ALGEBRAS
}

\author{
FABIO BAGARELLO, ATSUSHI INOUE, AND CAMILLO TRAPANI
}

(Communicated by Marius Junge)

\begin{abstract}
The unbounded bicommutant $\left(\mathfrak{M}_{E^{\prime}}\right)_{\text {wc }}^{\prime \prime}$ of the reduction of an $\mathrm{O}^{*}$ algebra $\mathfrak{M}$ via a given projection $E^{\prime}$ weakly commuting with $\mathfrak{M}$ is studied, with the aim of finding conditions under which the reduction of a $\mathrm{GW}^{*}$-algebra is a $\mathrm{GW}^{*}$-algebra itself. The obtained results are applied to the problem of the existence of conditional expectations on $\mathrm{O}^{*}$-algebras.
\end{abstract}

\section{INTRODUCTION AND PRELIMINARIES}

If $\mathfrak{M}$ is a von Neumann algebra and $E^{\prime}$ a projection on the commutant $\mathfrak{M}^{\prime}$ of $\mathfrak{M}$, then it is well known that the reduced algebra $\mathfrak{M}_{E^{\prime}}=\left\{X E^{\prime}: X \in \mathfrak{M}\right\}$ is again a von Neumann algebra. The notion of a von Neumann algebra has been generalized to unbounded operator algebras by introducing several classes of $\mathrm{O}^{*}$-algebras, such as $\mathrm{GW}^{*}$-algebras, $\mathrm{EW}^{*}$-algebras, etc. It is then natural and important for applications to pose the question if the reduced algebra of a $\mathrm{GW}^{*}$-algebra via a projection $E^{\prime}$ picked in its weak bounded commutant is again a $\mathrm{GW}^{*}$-algebra. In order to answer this question we undertake a more general study of the reduction process for $\mathrm{O}^{*}$-algebras. We begin with reviewing the basic definitions and properties of $\mathrm{O}^{*}$-algebras needed in this paper and refer to [5, 6, for more details.

Let $\mathcal{H}$ be a Hilbert space with inner product $\langle\cdot \mid \cdot\rangle$ and $\mathcal{D}$ a dense subspace of $\mathcal{H}$. We denote by $\mathcal{L}^{\dagger}(\mathcal{D})$ the set of all linear operators $X$ defined in $\mathcal{D}$ such that $X \mathcal{D} \subset \mathcal{D}$, the domain $D\left(X^{*}\right)$ of its adjoint contains $\mathcal{D}$ and $X^{*} \mathcal{D} \subset \mathcal{D}$. Then $\mathcal{L}^{\dagger}(\mathcal{D})$ is a *algebra with the usual operations: $X+Y, \alpha X, X Y$ and the involution $X \mapsto X^{\dagger}:=X^{*} \mid \mathcal{D}$. A *-subalgebra of $\mathcal{L}^{\dagger}(\mathcal{D})$ is called an $\mathrm{O}^{*}$-algebra on $\mathcal{D}$. In this paper we will assume that an $\mathrm{O}^{*}$-algebra always contains the identity $I$.

Let $\mathfrak{M}$ be an $\mathrm{O}^{*}$-algebra on $\mathcal{D}$. The graph topology $t_{\mathfrak{M}}$ on $\mathcal{D}$ is the locally convex topology defined by the family $\left\{\|\cdot\|_{X}: X \in \mathfrak{M}\right\}$ of seminorms: $\|\xi\|_{X}=\|X \xi\|$, $\xi \in \mathcal{D}$. If the locally convex space $\mathcal{D}\left[t_{\mathfrak{M}}\right]$ is complete, then $\mathfrak{M}$ is said to be closed. In particular, if $\mathcal{D}\left[t_{\mathfrak{M}}\right]$ is a Fréchet space, then $\mathcal{D}$ is called the Fréchet domain of $\mathfrak{M}$. More in general, we denote by $\widetilde{\mathcal{D}}(\mathfrak{M})$ the completion of the locally convex space $\mathcal{D}\left[t_{\mathfrak{M}}\right]$ and put

$$
\widetilde{X}:=\bar{X} \mid \widetilde{\mathcal{D}}(\mathfrak{M}) \quad \text { and } \widetilde{\mathfrak{M}}:=\{\widetilde{X}: X \in \mathfrak{M}\}
$$

Received by the editors September 9, 2008.

2000 Mathematics Subject Classification. Primary 47L60.

This work was supported by CORI, Università di Palermo. 
Then $\widetilde{\mathcal{D}}(\mathfrak{M})=\bigcap_{X \in \mathfrak{M}} D(\bar{X})$ and $\widetilde{\mathfrak{M}}$ is a closed $\mathrm{O}^{*}$-algebra on $\widetilde{\mathcal{D}}(\mathfrak{M})$ which is called the closure of $\mathfrak{M}$, since it is the smallest closed extension of $\mathfrak{M}$. We next recall the notion of self-adjointness of $\mathfrak{M}$. If $\mathcal{D}=\mathcal{D}^{*}(\mathfrak{M}):=\bigcap_{X \in \mathfrak{M}} D\left(X^{*}\right)$, then $\mathfrak{M}$ is said to be self-adjoint. If $\widetilde{\mathcal{D}}(\mathfrak{M})=\mathcal{D}^{*}(\mathfrak{M})$, then $\mathfrak{M}$ is said to be essentially self-adjoint. It is clear that

$$
\begin{aligned}
& \mathcal{D} \subset \widetilde{\mathcal{D}}(\mathfrak{M}) \subset \mathcal{D}^{*}(\mathfrak{M}), \\
& X \subset \widetilde{X} \subset X^{\dagger^{*}}, \quad \forall X \in \mathfrak{M} .
\end{aligned}
$$

The weak commutant $\mathfrak{M}_{\mathrm{w}}^{\prime}$ of $\mathfrak{M}$ is defined by

$$
\mathfrak{M}_{\mathrm{w}}^{\prime}=\left\{C \in \mathcal{B}(\mathcal{H}):\langle C X \xi \mid \eta\rangle=\left\langle C \xi \mid X^{\dagger} \eta\right\rangle, \forall X \in \mathfrak{M}, \forall \xi, \eta \in \mathcal{D}\right\},
$$

where $\mathcal{B}(\mathcal{H})$ is the ${ }^{*}$-algebra of all bounded linear operators on $\mathcal{H}$. Then $\mathfrak{M}_{\mathrm{w}}^{\prime}$ is a weak-operator closed ${ }^{*}$-invariant subspace of $\mathcal{B}(\mathcal{H})$ (but it is not, in general, a von Neumann algebra) and $(\widetilde{\mathfrak{M}})_{\mathrm{w}}^{\prime}=\mathfrak{M}_{\mathrm{w}}^{\prime}$. If $\mathfrak{M}_{\mathrm{w}}^{\prime} \mathcal{D} \subset \mathcal{D}$, as happens for self-adjoint $\mathfrak{M}$, then $\mathfrak{M}_{\mathrm{w}}^{\prime}$ is a von Neumann algebra. In this paper we will use the following bicommutants of $\mathfrak{M}$ :

$$
\begin{gathered}
\left(\mathfrak{M}_{\mathrm{w}}^{\prime}\right)^{\prime}=\left\{A \in \mathcal{B}(\mathcal{H}): A C=C A, \forall C \in \mathfrak{M}_{\mathrm{w}}^{\prime}\right\} \\
\mathfrak{M}_{\mathrm{wc}}^{\prime \prime}:=\left(\mathfrak{M}_{\mathrm{w}}^{\prime}\right)_{\mathrm{c}}^{\prime}=\left\{X \in \mathcal{L}^{\dagger}(\mathcal{D}):\langle C X \xi \mid \eta\rangle=\left\langle C \xi \mid X^{\dagger} \eta\right\rangle, \forall C \in \mathfrak{M}_{\mathrm{w}}^{\prime}, \forall \xi, \eta \in \mathcal{D}\right\} .
\end{gathered}
$$

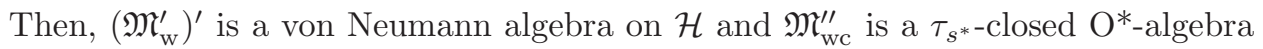
on $\mathcal{D}$ such that $\mathfrak{M} \subset \mathfrak{M}_{\mathrm{wc}}^{\prime \prime}$ and $\left(\mathfrak{M}_{\mathrm{wc}}^{\prime \prime}\right)_{\mathrm{w}}^{\prime}=\mathfrak{M}_{\mathrm{w}}^{\prime}$, where the strong*-topology $\tau_{s^{*}}$ is defined by the family $\left\{p_{\xi}^{*}(\cdot): \xi \in \mathcal{D}\right\}$ of seminorms:

$$
p_{\xi}^{*}(X):=\|X \xi\|+\left\|X^{\dagger} \xi\right\|, \quad X \in \mathcal{L}^{\dagger}(\mathcal{D}) .
$$

Furthermore, if $\mathfrak{M}_{\mathrm{w}}^{\prime} \mathcal{D} \subset \mathcal{D}$, then

$$
\mathfrak{M}_{\mathrm{wc}}^{\prime \prime}=\left\{X \in \mathcal{L}^{\dagger}(\mathcal{D}): \bar{X} \text { is affiliated with }\left(\mathfrak{M}_{\mathrm{w}}^{\prime}\right)^{\prime}\right\}=\overline{\left(\mathfrak{M}_{\mathrm{w}}^{\prime}\right)^{\prime}\lceil\mathcal{D}}{ }^{\tau_{s^{*}}} \cap \mathcal{L}^{\dagger}(\mathcal{D}) .
$$

An $\mathrm{O}^{*}$-algebra $\mathfrak{M}$ on $\mathcal{D}$ is called a $G W^{*}$-algebra if $\mathfrak{M}_{\mathrm{w}}^{\prime} \mathcal{D} \subset \mathcal{D}$ and $\mathfrak{M}=\mathfrak{M}_{\mathrm{wc}}^{\prime \prime}$.

Let $\mathfrak{M}$ be a closed $\mathrm{O}^{*}$-algebra on $\mathcal{D}$ such that $\mathfrak{M}_{\mathrm{w}}^{\prime} \mathcal{D} \subset \mathcal{D}$ and let $E^{\prime}$ be a projection in the von Neumann algebra $\mathfrak{M}_{\mathrm{w}}^{\prime}$. Let $\mathfrak{M}_{E^{\prime}}$ denote the reduced algebra $\mathfrak{M}_{E^{\prime}}=\left\{X E^{\prime}: X \in \mathfrak{M}\right\}$. Then it is known [1] that

$$
\begin{aligned}
& \left(\mathfrak{M}_{E^{\prime}}\right)_{\mathrm{w}}^{\prime}=\left(\mathfrak{M}_{\mathrm{w}}^{\prime}\right)_{E^{\prime}}, \\
& \left(\left(\mathfrak{M}_{E^{\prime}}\right)_{\mathrm{w}}^{\prime}\right)^{\prime}=\left(\left(\mathfrak{M}_{\mathrm{w}}^{\prime}\right)^{\prime}\right)_{E^{\prime}}
\end{aligned}
$$

But the following question is open.

Question. Does the equality $\left(\mathfrak{M}_{E^{\prime}}\right)_{\mathrm{wc}}^{\prime \prime}=\left(\mathfrak{M}_{\mathrm{wc}}^{\prime \prime}\right)_{E^{\prime}}$ hold?

If the answer to this question is affirmative, then the reduced algebra $\mathfrak{M}_{E^{\prime}}$ of a given $\mathrm{GW}^{*}$-algebra is again a $\mathrm{GW}^{*}$-algebra. This question will be considered in Section 2, where we will show that if $\mathcal{D}$ is the Fréchet domain of $\mathfrak{M}$ and the linear span $\left\langle\mathfrak{M}_{\mathrm{w}}^{\prime} E^{\prime} \mathcal{D}\right\rangle$ of the $\mathfrak{M}$-invariant subspace $\mathfrak{M}_{\mathrm{w}}^{\prime} E^{\prime} \mathcal{D}$ is essentially self-adjoint, then $\left(\mathfrak{M}_{E^{\prime}}\right)_{\mathrm{wc}}^{\prime \prime}=\left(\mathfrak{M}_{\mathrm{wc}}^{\prime \prime}\right)_{E^{\prime}}$. An $\mathfrak{M}$-invariant subspace $\mathcal{M}$ of $\mathcal{D}$ is called essentially selfadjoint if the $\mathrm{O}^{*}$-algebra $\mathfrak{M}\lceil\mathcal{M}:=\{X \mid \mathcal{M}: X \in \mathfrak{M}\}$ of $\mathcal{M}$ is essentially selfadjoint.

Furthermore, we will show that, if the graph topology $t_{\mathfrak{M}}$ is defined by a sequence $\left\{T_{n}\right\}$ of essentially self-adjoint operators of $\mathfrak{M}$, whose spectral projections leave the domain $\mathcal{D}$ invariant, then again $\left(\mathfrak{M}_{E^{\prime}}\right)_{\mathrm{wc}}^{\prime \prime}=\left(\mathfrak{M}_{\mathrm{wc}}^{\prime \prime}\right)_{E^{\prime}}$. 
In Section 3 we shall apply the results of Section 2 to the study of conditional expectations for $\mathrm{O}^{*}$-algebras.

\section{MAin RESUlts}

Let $\mathfrak{M}$ be a closed $\mathrm{O}^{*}$-algebra on $\mathcal{D}$ such that $\mathfrak{M}_{\mathrm{w}}^{\prime} \mathcal{D} \subset \mathcal{D}$ and let $E^{\prime}$ be a projection in the von Neumann algebra $\mathfrak{M}_{\mathrm{w}}^{\prime}$. In this section we look for conditions for the equality $\left(\mathfrak{M}_{\mathrm{wc}}^{\prime \prime}\right)_{E^{\prime}}=\left(\mathfrak{M}_{E^{\prime}}\right)_{\mathrm{wc}}^{\prime \prime}$ to hold. Since $\left(\mathfrak{M}_{E^{\prime}}\right)_{\mathrm{w}}^{\prime}=\left(\mathfrak{M}_{\mathrm{w}}^{\prime}\right)_{E^{\prime}}$, it follows that

$$
\left(\mathfrak{M}_{\mathrm{wc}}^{\prime \prime}\right)_{E^{\prime}} \subset\left(\left(\mathfrak{M}_{\mathrm{w}}^{\prime}\right)_{E^{\prime}}\right)_{\mathrm{c}}^{\prime}=\left(\mathfrak{M}_{E^{\prime}}\right)_{\mathrm{wc}}^{\prime \prime} .
$$

Hence we need only to prove the converse inclusion: $\left(\mathfrak{M}_{E^{\prime}}\right)_{\mathrm{wc}}^{\prime \prime} \subset\left(\mathfrak{M}_{\mathrm{wc}}^{\prime \prime}\right)_{E^{\prime}}$. An element $X$ of $\left(\mathfrak{M}_{E^{\prime}}\right)_{\mathrm{wc}}^{\prime \prime}$ is an operator on $E^{\prime} \mathcal{D}$, and so to show that $X \in\left(\mathfrak{M}_{\mathrm{wc}}^{\prime \prime}\right)_{E^{\prime}}$, we need to extend $X$ to an operator on $\mathcal{D}$. We will consider this extension problem below.

Let $Z$ denote the central support of $E^{\prime}$; i.e., $Z$ is the projection onto the closure of the subspace $\left\langle\mathfrak{M}_{\mathrm{w}}^{\prime} E^{\prime} \mathcal{H}\right\rangle$. We define $\mathcal{E}:=\left\langle\mathfrak{M}_{\mathrm{w}}^{\prime} E^{\prime} \mathcal{D}\right\rangle \oplus(I-Z) \mathcal{D}$. Then $\left\langle\mathfrak{M}_{\mathrm{w}}^{\prime} E^{\prime} \mathcal{D}\right\rangle \subset$ $Z D$ and $\mathcal{E} \subset \mathcal{D}$.

For $X \in\left(\mathfrak{M}_{E^{\prime}}\right)_{\mathrm{wc}}^{\prime \prime}$, we put

$$
X_{e}\left(\sum_{k} C_{k} E^{\prime} \xi_{k}+(I-Z) \eta\right):=\sum_{k} C_{k} X E^{\prime} \xi_{k}, \quad C_{k} \in \mathfrak{M}_{\mathrm{w}}^{\prime}, \xi_{k}, \eta \in \mathcal{D} .
$$

Then we have the following.

Lemma 2.1. $X_{e} \in \mathcal{L}^{\dagger}(\mathcal{E})$ and $\left(\left(\mathfrak{M}_{E^{\prime}}\right)_{\mathrm{wc}}^{\prime \prime}\right)_{e}:=\left\{X_{e}: X \in\left(\mathfrak{M}_{E^{\prime}}\right)_{\mathrm{wc}}^{\prime \prime}\right\}$ is an $O^{*}$-algebra on $\mathcal{E}$.

Proof. For any $X \in\left(\mathfrak{M}_{E^{\prime}}\right)_{\mathrm{wc}}^{\prime \prime}, C_{k}, K_{j} \in \mathfrak{M}_{\mathrm{w}}^{\prime}$ and $\xi_{k}, \zeta_{j}, \eta, \eta_{1} \in \mathcal{D}$ we have

$$
\begin{aligned}
& \left\langle X_{e}\left(\sum_{k} C_{k} E^{\prime} \xi_{k}+(I-Z) \eta\right) \mid \sum_{j} K_{j} E^{\prime} \zeta_{j}+(I-Z) \eta_{1}\right\rangle \\
& =\sum_{k, j}\left\langle C_{k} X E^{\prime} \xi_{k} \mid K_{j} E^{\prime} \zeta_{j}\right\rangle \\
& =\sum_{k, j}\left\langle E^{\prime} K_{j}^{*} C_{k} E^{\prime} X E^{\prime} \xi_{k} \mid E^{\prime} \zeta_{j}\right\rangle \\
& =\sum_{k, j}\left\langle E^{\prime} K_{j}^{*} C_{k} E^{\prime} \xi_{k} \mid X^{\dagger} E^{\prime} \zeta_{j}\right\rangle \\
& =\left\langle\sum_{k} C_{k} E^{\prime} \xi_{k} \mid \sum_{j} K_{j} X^{\dagger} E^{\prime} \zeta_{j}\right\rangle \\
& =\left\langle\sum_{k} C_{k} E^{\prime} \xi_{k}+(I-Z) \eta \mid\left(X_{e}\right)^{\dagger}\left(\sum_{j} K_{j} E^{\prime} \zeta_{j}+(I-Z) \eta_{1}\right)\right\rangle,
\end{aligned}
$$

which implies that $X_{e}$ is well-defined and $\left(X_{e}\right)^{\dagger}=\left(X^{\dagger}\right)_{e}$. It is clear that $X_{e}$ is a linear operator on $\mathcal{E}$. Hence $X_{e} \in \mathcal{L}^{\dagger}(\mathcal{E})$. Moreover, as is easily seen, $(X Y)_{e}=$ $X_{e} Y_{e}$. Hence, $e\left(\mathfrak{M}_{E^{\prime}}\right):=\left(\left(\mathfrak{M}_{E^{\prime}}\right)_{\text {wc }}^{\prime \prime}\right)_{e}$ is an $\mathrm{O}^{*}$-algebra on $\mathcal{E}$. 
Since $e\left(\mathfrak{M}_{E^{\prime}}\right)$ is an $\mathrm{O}^{*}$-algebra on $\mathcal{E}$, its closure is an $\mathrm{O}^{*}$-algebra on $\widetilde{\mathcal{E}}$. It is easily

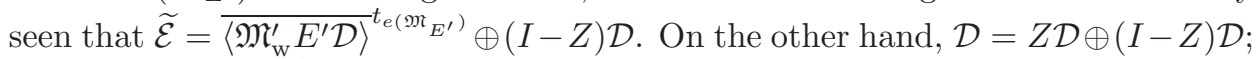
hence, if $\overline{\left\langle\mathfrak{M}_{\mathrm{w}}^{\prime} E^{\prime} \mathcal{D}\right\rangle^{t}} t_{e\left(\mathfrak{M}_{E^{\prime}}\right)}=Z \mathcal{D}$, we can extend any $X \in\left(\mathfrak{M}_{E^{\prime}}\right)_{\mathrm{wc}}^{\prime \prime}$ to $\mathcal{D}$.

Theorem 2.2. Let $\mathfrak{M}$ be a closed $O^{*}$-algebra on $\mathcal{D}$ such that $\mathfrak{M}_{\mathrm{w}}^{\prime} \mathcal{D} \subset \mathcal{D}$ and let $E^{\prime}$ be a projection in the von Neumann algebra $\mathfrak{M}_{\mathrm{w}}^{\prime}$. Suppose that $\left.\widetilde{\mathcal{E}}\left(\left(\mathfrak{M}_{E^{\prime}}\right)_{\mathrm{wc}}^{\prime \prime}\right)_{e}\right)=\mathcal{D}$ or, equivalently, $\overline{\left\langle\mathfrak{M}_{\mathrm{w}}^{\prime} E^{\prime} \mathcal{D}\right\rangle} t_{e\left(\mathfrak{M}_{E^{\prime}}\right)}=Z \mathcal{D}$. Then, $\left(\mathfrak{M}_{\mathrm{wc}}^{\prime \prime}\right)_{E^{\prime}}=\left(\mathfrak{M}_{E^{\prime}}\right)_{\mathrm{wc}}^{\prime \prime}$.

Proof. By the assumption, for every $X \in\left(\mathfrak{M}_{E^{\prime}}\right)_{\mathrm{wc}}^{\prime \prime}, X_{e}$ extends to an operator $\widetilde{X_{e}}$ on $\mathcal{D}$; that is, $\widetilde{X_{e}}=\overline{X_{e}}\left\lceil\mathcal{D}\right.$ and $\left(\widetilde{X_{e}}\right)_{E^{\prime}}=X$. Furthermore, we have $\widetilde{X_{e}} \in \mathfrak{M}_{\mathrm{wc}}^{\prime \prime}$. Indeed, for every $C \in \mathfrak{M}_{\mathrm{w}}^{\prime}$, we have

$$
\begin{aligned}
C X_{e}\left(\sum_{k} C_{k} E^{\prime} \xi_{k}+(I-Z) \eta\right) & =C \sum_{k} C_{k} X E^{\prime} \xi_{k} \\
& =\sum_{k} C C_{k} X E^{\prime} \xi_{k} \\
& =X_{e} C\left(\sum_{k} C_{k} E^{\prime} \xi_{k}+(I-Z) \eta\right),
\end{aligned}
$$

for every $C_{k} \in \mathfrak{M}_{\mathrm{w}}^{\prime}, \xi_{k}, \eta \in \mathcal{D}$. Hence, $C X_{e}=X_{e} C$ on $\mathcal{E}$ and so $C \widetilde{X_{e}}=\widetilde{X_{e}} C$. Thus $\widetilde{X_{e}} \in \mathfrak{M}_{\mathrm{wc}}^{\prime \prime}$ and $X=\left(\widetilde{X_{e}}\right)_{E^{\prime}} \in\left(\mathfrak{M}_{\mathrm{wc}}^{\prime \prime}\right)_{E^{\prime}}$. In conclusion, $\left(\mathfrak{M}_{E^{\prime}}\right)_{\mathrm{wc}}^{\prime \prime} \subset\left(\mathfrak{M}_{\mathrm{wc}}^{\prime \prime}\right)_{E^{\prime}}$ and by (11) the equality follows.

The next step consists, of course, in looking for situations where the conditions of Theorem 2.2 are satisfied. We begin with the following.

Lemma 2.3. Let $\mathfrak{M}$ and $E^{\prime}$ be as above. Suppose that

(i) for every $X \in\left(\mathfrak{M}_{E^{\prime}}\right)_{\mathrm{wc}}^{\prime \prime}$ there exists an element $Y$ of $\mathfrak{M}_{\mathrm{wc}}^{\prime \prime}$ such that

$$
\left\|X_{e} \xi\right\| \leq\|Y \xi\|, \quad \forall \xi \in \mathcal{E}
$$

(equivalently, $X_{e}^{\dagger} X_{e} \leq Y^{\dagger} Y$ on $\mathcal{E}$ );

(ii) $\overline{\left\langle\mathfrak{M}_{\mathrm{w}}^{\prime} E^{\prime} \mathcal{D}\right\rangle}{ }^{t_{\mathfrak{M}}{ }_{\mathrm{wc}}^{\prime \prime}}=Z \mathcal{D}$.

Then, $\left(\mathfrak{M}_{E^{\prime}}\right)_{\mathrm{wc}}^{\prime \prime}=\left(\mathfrak{M}_{\mathrm{wc}}^{\prime \prime}\right)_{E^{\prime}}$.

Proof. The conditions (i) and (ii) immediately imply that

$$
Z \mathcal{D} \subset{\overline{\left\langle\mathfrak{M}_{\mathrm{w}}^{\prime} E^{\prime} \mathcal{D}\right\rangle}}^{t_{e\left(\mathfrak{M}_{E^{\prime}}\right)}} .
$$

Hence

$$
{\overline{\left\langle\mathfrak{M}_{\mathrm{w}}^{\prime} E^{\prime} \mathcal{D}\right\rangle}}^{t_{e\left(\mathfrak{M}_{E^{\prime}}\right)}}=Z \mathcal{D} \text {. }
$$

By Theorem 2.2, we get $\left(\mathfrak{M}_{E^{\prime}}\right)_{\mathrm{wc}}^{\prime \prime}=\left(\mathfrak{M}_{\mathrm{wc}}^{\prime \prime}\right)_{E^{\prime}}$.

Remark 2.4. A comment is in order for condition (ii) of Lemma 2.3, Let us in fact consider the following statements:

(i) The $\mathfrak{M}_{\mathrm{wc}}^{\prime \prime}$-invariant subspace $\left\langle\mathfrak{M}_{\mathrm{w}}^{\prime} E^{\prime} \mathcal{D}\right\rangle$ is essentially self-adjoint for $\mathfrak{M}_{\mathrm{wc}}^{\prime \prime}$.

(ii) ${\overline{\left\langle\mathfrak{M}_{\mathrm{w}}^{\prime} E^{\prime} \mathcal{D}\right\rangle}}^{t_{\mathfrak{M} \mathrm{wc}}^{\prime \prime}}=Z \mathcal{D}$.

Then (i) $\Rightarrow$ (ii). In particular if $\mathfrak{M}$ is self-adjoint, then the two conditions are equivalent. For the proof we refer to [5]. 
Theorem 2.5. Let $\mathfrak{M}$ be a closed $O^{*}$-algebra $\mathcal{D}$ with $\mathcal{D}$ a Fréchet domain of $\mathfrak{M}$. Assume that $\mathfrak{M}_{\mathrm{w}}^{\prime} \mathcal{D} \subset \mathcal{D}$. Let $E^{\prime}$ be a projection in $\mathfrak{M}_{\mathrm{w}}^{\prime}$ and assume that $\left\langle\mathfrak{M}_{\mathrm{w}}^{\prime} E^{\prime} \mathcal{D}\right\rangle$ is essentially self-adjoint for $\mathfrak{M}_{\mathrm{wc}}^{\prime \prime}$. Then, $\left(\mathfrak{M}_{E^{\prime}}\right)_{\mathrm{wc}}^{\prime \prime}=\left(\mathfrak{M}_{\mathrm{wc}}^{\prime \prime}\right)_{E^{\prime}}$.

Proof. Since $\mathcal{D}\left[t_{\mathfrak{M}}\right]$ is a Fréchet space, the topology $t_{\mathfrak{M}}$ is defined by a sequence $\left\{T_{n}\right\}$ of elements of $\mathfrak{M}$ satisfying

$$
\|\xi\| \leq\left\|T_{1} \xi\right\| \leq\left\|T_{2} \xi\right\|, \ldots, \quad \forall \xi \in \mathcal{D} .
$$

Any $X \in \mathcal{L}^{\dagger}(\mathcal{D})$ is a closed linear operator on the Fréchet space $\mathcal{D}\left[t_{\mathfrak{M}}\right]$ into the Hilbert space $\mathcal{H}$, which implies, by the closed graph theorem, that the topology $t_{\mathfrak{M}}$ is equivalent to the graph topology $t_{\mathcal{L}^{\dagger}(\mathcal{D})}$ defined by $\mathcal{L}^{\dagger}(\mathcal{D})$. This in turn implies that for every $X \in \mathcal{L}^{\dagger}(\mathcal{D})$ there exist $n_{0} \in \mathbb{N}$ and $\gamma>0$ such that

$$
\|X \xi\| \leq \gamma\left\|T_{n_{0}} \xi\right\|, \quad \forall \xi \in \mathcal{D} .
$$

Now take an arbitrary $X \in\left(\mathfrak{M}_{E^{\prime}}\right)_{\mathrm{wc}}^{\prime \prime}$. Since $X E^{\prime} \in \mathcal{L}^{\dagger}(\mathcal{D})$, it follows from (2) that

$$
\left\|X E^{\prime} \xi\right\| \leq \gamma\left\|T_{n_{0}} \xi\right\|, \quad \forall \xi \in \mathcal{D} .
$$

Since $\mathfrak{M}_{\mathrm{w}}^{\prime}$ is a von Neumann algebra, for every $C \in \mathfrak{M}_{\mathrm{w}}^{\prime}$ and $\xi \in \mathcal{D}$, we have

$$
\begin{aligned}
\left\|X_{e}\left(C E^{\prime} \xi\right)\right\|^{2} & =\left\|C X E^{\prime} \xi\right\|^{2} \\
& =\left\langle E^{\prime} C^{*} C E^{\prime} X E^{\prime} \xi \mid X E^{\prime} \xi\right\rangle \\
& =\left\|\left(E^{\prime} C^{*} C E^{\prime}\right)^{1 / 2} X E^{\prime} \xi\right\|^{2} \\
& =\left\|X E^{\prime}\left(E^{\prime} C^{*} C E^{\prime}\right)^{1 / 2} \xi\right\|^{2} \\
& \leq \gamma^{2}\left\|T_{n_{0}}\left(E^{\prime} C^{*} C E^{\prime}\right)^{1 / 2} \xi\right\|^{2} \\
& =\gamma^{2}\left\|\left(E^{\prime} C^{*} C E^{\prime}\right)^{1 / 2} T_{n_{0}} \xi\right\|^{2} \\
& =\gamma^{2}\left\|C E^{\prime} T_{n_{0}} \xi\right\|^{2} \\
& =\gamma^{2}\left\|T_{n_{0}} C E^{\prime} \xi\right\|^{2} .
\end{aligned}
$$

Hence, condition (i) of Lemma 2.3 is satisfied. By the same lemma we then get the equality $\left(\mathfrak{M}_{E^{\prime}}\right)_{\mathrm{wc}}^{\prime \prime}=\left(\mathfrak{M}_{\mathrm{wc}}^{\prime \prime}\right)_{E^{\prime}}$.

Lemma 2.6. Let $\mathfrak{M}$ be a closed $O^{*}$-algebra on a Fréchet domain $\mathcal{D}$ in $\mathcal{H}$ such that $t_{\mathfrak{M}}$ is defined by a sequence $\left\{T_{n}\right\}$ of essentially self-adjoint operators of $\mathfrak{M}$ whose spectral projections belong to $\mathfrak{M}$.

Let $\mathcal{M}$ be an $\mathfrak{M}$-invariant subspace of $\mathcal{D}$ such that the projection $P_{\mathcal{M}}$ onto $\overline{\mathcal{M}}$ belongs to $\mathfrak{M}_{w}^{\prime}$.

Then $\mathcal{M}$ is essentially self-adjoint for $\mathfrak{M}$ or, equivalently, $P_{\mathcal{M}} \mathcal{D}=\overline{\mathcal{M}}^{t_{\mathfrak{M}}}$.

Proof. First we observe that $\overline{\mathcal{M}}^{t_{\mathfrak{M}}} \subset P_{\mathcal{M}} \mathcal{D}$. Hence we only need to show that the converse inclusion also holds. Take an arbitrary $\xi \in \mathcal{D}$. Then there exists a sequence $\left\{\xi_{n}\right\}$ in $\mathcal{M}$ such that $\lim _{n \rightarrow \infty}\left\|\xi_{n}-P_{\mathcal{M}} \xi\right\|=0$. By (3), for every $X \in \mathfrak{M}$, there exists $n_{0} \in \mathbb{N}$ and $\gamma>0$ such that

$$
\|X \xi\| \leq \gamma\left\|T_{n_{0}} \xi\right\|, \quad \forall \xi \in \mathcal{D} .
$$

By the assumption, $\overline{T_{n_{0}}}$ is self-adjoint. Let $\overline{T_{n_{0}}}=\int_{-\infty}^{\infty} \lambda d E_{T_{n_{0}}}(\lambda)$ be the spectral resolution of $\overline{T_{n_{0}}}$. We put $E_{k}:=E_{T_{n_{0}}}(k)-E_{T_{n_{0}}}(-k), k \in \mathbb{N}$. Then, by assumption $E_{k} \mid \mathcal{D} \in \mathfrak{M}, \forall k \in \mathbb{N}$ and so, $E_{k} \xi_{n} \in \mathcal{M}, \forall k, n \in \mathbb{N}$ and $\lim _{n \rightarrow \infty}\left\|E_{k} \xi_{n}-E_{k} P_{\mathcal{M}} \xi\right\|=$ 0 . Furthermore, by (4) we have

$$
\left\|X E_{k} \xi_{n}-X E_{k} \xi_{m}\right\| \leq \gamma\left\|T_{n_{0}} E_{k}\left(\xi_{n}-\xi_{m}\right)\right\| \rightarrow 0, \text { as } n, m \rightarrow \infty .
$$


Hence $E_{k} P_{\mathcal{M}} \xi \in D(\overline{X\lceil\mathcal{M}})$.

Furthermore,

$$
\begin{aligned}
\left\|X E_{k} P_{\mathcal{M}} \xi-X P_{\mathcal{M}} \xi\right\| & \leq \gamma\left\|T_{n_{0}} E_{k} P_{\mathcal{M}} \xi-T_{n_{0}} P_{\mathcal{M}} \xi\right\| \\
& =\gamma\left\|P_{\mathcal{M}}\left(E_{k} T_{n_{0}} \xi-T_{n_{0}} \xi\right)\right\| \\
& \leq \gamma\left\|E_{k} T_{n_{0}} \xi-T_{n_{0}} \xi\right\| \rightarrow 0 \text { as } k \rightarrow \infty,
\end{aligned}
$$

which implies that $P_{\mathcal{M}} \xi \in D(\overline{X\lceil\mathcal{M}})$. Hence,

$$
P_{\mathcal{M}} \xi \in \bigcap_{X \in \mathfrak{M}} D(\overline{X\lceil\mathcal{M}})=\overline{\mathcal{M}}^{t_{\mathfrak{M}}}
$$

Thus $P_{\mathcal{M}} \mathcal{D}=\overline{\mathcal{M}}^{t_{\mathfrak{M}}}$. This is equivalent to $\mathcal{M}$ being essentially self-adjoint for $\mathfrak{M}$, since $\mathfrak{M}$ is clearly self-adjoint.

By Theorem 2.5 and Lemma 2.6 we have the following.

Theorem 2.7. Let $\mathfrak{M}$ be a closed $O^{*}$-algebra such that $t_{\mathfrak{M}}$ is defined by a sequence $\left\{T_{n}\right\}$ of essentially self-adjoint operators of $\mathfrak{M}$ whose spectral projections leave the domain $\mathcal{D}$ invariant. Let $E^{\prime}$ be a projection in $\mathfrak{M}_{\mathrm{w}}^{\prime}$. Then $\left(\mathfrak{M}_{E^{\prime}}\right)_{\mathrm{wc}}^{\prime \prime}=\left(\mathfrak{M}_{\mathrm{wc}}^{\prime \prime}\right)_{E^{\prime}}$.

Proof. It can be shown that $\mathfrak{M}$ is a self-adjoint $\mathrm{O}^{*}$-algebra on the Fréchet domain $\mathcal{D}$, so that $\mathfrak{M}_{w}^{\prime} \mathcal{D} \subset \mathcal{D}, t_{\mathfrak{M}_{\text {wc }}^{\prime \prime}}=t_{\mathfrak{M}}$ and $\mathfrak{M}_{\text {wc }}^{\prime \prime}$ is a self-adjoint $\mathrm{O}^{*}$-algebra on the Fréchet domain $\mathcal{D}$. Furthermore

$$
P_{\left\langle\mathcal{M}_{w}^{\prime} E^{\prime} \mathcal{D}\right\rangle}=Z \in \mathfrak{M}_{w}^{\prime} \cap\left(\mathfrak{M}_{w}^{\prime}\right)^{\prime}=\left(\mathfrak{M}_{\mathrm{wc}}^{\prime \prime}\right)_{\mathrm{w}}^{\prime} \cap\left(\left(\mathfrak{M}_{\mathrm{wc}}^{\prime \prime}\right)_{\mathrm{w}}^{\prime}\right)^{\prime} .
$$

Moreover, since the spectral projections of the operators $T_{n}$ leave the domain $\mathcal{D}$ invariant, they automatically belong to $\mathfrak{M}_{\mathrm{wc}}^{\prime \prime}$. Hence, the self-adjoint $\mathrm{O}^{*}$-algebra $\mathfrak{M}_{\mathrm{wc}}^{\prime \prime}$ and the $\mathfrak{M}_{\mathrm{wc}}^{\prime \prime}$-invariant subspace $\left\langle\mathcal{M}_{w}^{\prime} E^{\prime} \mathcal{D}\right\rangle$ of $\mathcal{D}$ satisfy all the conditions in

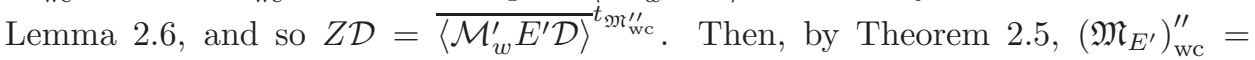
$\left(\mathfrak{M}_{\mathrm{wc}}^{\prime \prime}\right)_{E^{\prime}}$.

Corollary 2.8. Let $T$ be an essentially self-adjoint operator in $\mathcal{H}$ and $\mathfrak{M}$ be a selfadjoint $O^{*}$-algebra on $\mathcal{D}^{\infty}(\bar{T}):=\bigcap_{n \in \mathbb{N}} D\left(\bar{T}^{n}\right)$, containing $T$. Let $E^{\prime}$ be a projection in $\mathfrak{M}_{\mathrm{w}}^{\prime}$. Then $\left(\mathfrak{M}_{E^{\prime}}\right)_{\mathrm{wc}}^{\prime \prime}=\left(\mathfrak{M}_{\mathrm{wc}}^{\prime \prime}\right)_{E^{\prime}}$.

Proof. The spectral projections $E_{T}(\lambda), \lambda \in \mathbb{R}$, of $\bar{T}$ satisfy:

- $E_{T}(\lambda) \in\left(\mathfrak{M}_{\mathrm{w}}^{\prime}\right)^{\prime}, \quad \forall \lambda \in \mathbb{R}$;

- $E_{T}(\lambda) \mathcal{H} \subset \mathcal{D}^{\infty}(\bar{T}), \quad \forall \lambda \in \mathbb{R}$.

The statement then follows from Theorem 2.7 .

Apart from $\mathrm{GW}^{*}$-algebras, another unbounded generalization of von Neumann algebras is provided by the notion of extended $\mathrm{W}^{*}$-algebras, shortly $\mathrm{EW}^{*}$-algebras, defined as follows: A closed $\mathrm{O}^{*}$-algebra $\mathfrak{M}$ on $\mathcal{D}$ is said to be an $E W^{*}$-algebra if $\left(I+X^{\dagger} X\right)^{-1}$ exists in $\mathfrak{M}_{\mathrm{b}}:=\{A \in \mathfrak{M}: \bar{A} \in \mathcal{B}(\mathcal{H})\}$, for every $X \in \mathfrak{M}$ and $\overline{\mathfrak{M}_{\mathrm{b}}}:=\left\{\bar{A} \in \mathfrak{M}: A \in \mathfrak{M}_{\mathrm{b}}\right\}$ is a von Neumann algebra 2. It is easily shown that every $\mathrm{EW}^{*}$-algebra on a Fréchet domain satisfies the conditions of Theorem 2.7 (recall that every symmetric element of an $\mathrm{EW}^{*}$-algebra is essentially self-adjoint). Hence we have the following.

Corollary 2.9. Let $\mathfrak{M}$ be an $E W^{*}$-algebra on the Fréchet domain $\mathcal{D}$ in Hilbert space $\mathcal{H}$ and $E^{\prime}$ a projection in $\mathfrak{M}_{\mathrm{w}}^{\prime}$. Then, $\left(\mathfrak{M}_{E^{\prime}}\right)_{\mathrm{wc}}^{\prime \prime}=\left(\mathfrak{M}_{\mathrm{wc}}^{\prime \prime}\right)_{E^{\prime}}$. 


\section{Applications}

In this section we show how to use the results of Section 2 in the analysis of the existence of conditional expectations for $\mathrm{O}^{*}$-algebras, which were first studied in [3, 4].

Let $\mathfrak{M}$ be a given $\mathrm{O}^{*}$-algebra on $\mathcal{D}$ in $\mathcal{H}$ with a strongly cyclic vector $\xi_{0}$. Here $\xi_{0} \in \mathcal{D}$ is said to be strongly cyclic for $\mathfrak{M}$ if $\overline{\mathfrak{M}}_{0}^{t_{\mathfrak{M}}}=\mathcal{D}$. With no loss of generality we will assume that $\left\|\xi_{0}\right\|=1$. Let $\mathfrak{N}$ be an $\mathrm{O}^{*}$-subalgebra of $\mathfrak{M}$. A map $\mathcal{E}$ of $\mathfrak{M}$ onto $\mathfrak{N}$ is said to be a conditional expectation of $\left(\mathfrak{M}, \xi_{0}\right)$ w.r.t. $\mathfrak{N}$ if it satisfies the following conditions:

(i) $\mathcal{E}(X)^{\dagger}=\mathcal{E}\left(X^{\dagger}\right), \forall X \in \mathfrak{M}$, and $\mathcal{E}(A)=A, \forall A \in \mathfrak{N}$;

(ii) $\mathcal{E}(X A)=\mathcal{E}(X) A$ and $\mathcal{E}(A X)=A \mathcal{E}(X), \forall X \in \mathfrak{M}, \forall A \in \mathfrak{N}$;

(iii) $\omega_{\xi_{0}}(\mathcal{E}(X))=\omega_{\xi_{0}}(X), \forall X \in \mathfrak{M}$, where $\omega_{\xi_{0}}$ is a state on $\mathfrak{M}$ defined as $\omega_{\xi_{0}}(X)=\left\langle X \xi_{0} \mid \xi_{0}\right\rangle, X \in \mathfrak{M}$.

In the case of von Neumann algebras, Takesaki 7] characterized the existence of conditional expectations using Tomita's modular theory. Thus a conditional expectation does not necessarily exist for a general von Neumann algebra. Hence, in [3, 4, Ogi, Takakura and the second author considered a linear map $\mathcal{E}$ of a †invariant subspace $D(\mathcal{E})$ of $\mathfrak{M}$ onto $\mathfrak{N}$ satisfying the above conditions (i), (ii) and (iii) on $D(\mathcal{E})$. Such a map is called an unbounded conditional expectation of $\left(\mathfrak{M}, \xi_{0}\right)$ w.r.t. $\mathfrak{N}$, and it was shown that there exists the largest unbounded conditional expectation $\mathcal{E}_{\mathfrak{N}}$ of $\left(\mathfrak{M}, \xi_{0}\right)$ w.r.t. $\mathfrak{N}$. Furthermore, the existence of conditional expectation was characterized, using Takesaki's result in the case of von Neumann algebras. But a deeper analysis is needed since, at that stage, the problem was not solved even in the case of $\mathrm{GW}^{*}$-algebras. One of the reasons is that the reduction of a $\mathrm{GW}^{*}$-algebra is not necessarily a $\mathrm{GW}^{*}$-algebra, in contrast with the case of von Neumann algebras. For $\mathcal{E}_{\mathfrak{N}}$ to be a conditional expectation of $\left(\mathfrak{M}, \xi_{0}\right)$ w.r.t. $\mathfrak{N}$ (i.e., everywhere defined on $\mathfrak{M}$ ), the following result given in [4, Corollary 6.2] holds.

Lemma 3.1. Let $\mathfrak{M}$ be a closed $O^{*}$-algebra on $\mathcal{D}$ in $\mathcal{H}$ such that $\mathfrak{M}_{w}^{\prime} \mathcal{D} \subset \mathcal{D}$. Let $\xi_{0}$ be a strongly cyclic and separating vector, in the sense that $\overline{\mathfrak{M}_{w}^{\prime} \xi_{0}}=\mathcal{H}$. Suppose that $\mathfrak{N}$ is a closed $O^{*}$-subalgebra of $\mathfrak{M}$ satisfying

(i) $\mathfrak{N}_{w}^{\prime} \mathcal{D} \subset \mathcal{D}$;

(ii) $\mathfrak{N} \xi_{0}$ is essentially self-adjoint for $\mathfrak{N}$;

(iii) $\Delta_{\xi_{0}}^{\prime \prime}\left(\mathfrak{N}_{w}^{\prime}\right)^{\prime} \Delta_{\xi_{0}}^{\prime \prime}{ }^{-i t}=\left(\mathfrak{N}_{w}^{\prime}\right)^{\prime}, \forall t \in \mathbb{R}$, where $\Delta_{\xi_{0}}^{\prime \prime}$ is the modular operator of the left Hilbert algebra $\left(\mathfrak{M}_{w}^{\prime}\right)^{\prime} \xi_{0}$;

(iv) $\mathfrak{N}_{P_{\mathfrak{N}}}$ is a $G W^{*}$-algebra on $P_{\mathfrak{N}} \mathcal{D}$, where $P_{\mathfrak{N}}$ is a projection of $\mathcal{H}$ onto $\overline{\mathfrak{N} \xi_{0}}$.

Then $\mathcal{E}_{\mathfrak{N}}$ is a conditional expectation of $\left(\mathfrak{M}, \xi_{0}\right)$ w.r.t. $\mathfrak{N}$.

By Lemma 3.1 and Theorem 2.5 we deduce the following.

Theorem 3.2. Let $\mathfrak{M}$ be a closed $O^{*}$-algebra on $\mathcal{D}$ in $\mathcal{H}$ with a strongly cyclic and separating vector $\xi_{0}$ such that $\mathfrak{M}_{w}^{\prime} \mathcal{D} \subset \mathcal{D}$ and let $\mathfrak{N}$ be an $O^{*}$-subalgebra of $\mathfrak{M}$.

Suppose that $\mathfrak{N}$ is a $G W^{*}$-algebra on a Fréchet domain $\mathcal{D}$ satisfying

(i) $\mathfrak{N} \xi_{0}$ and $\left\langle\mathfrak{N}_{w}^{\prime} P_{\mathfrak{N}} \mathcal{D}\right\rangle$ are essentially self-adjoint for $\mathfrak{N}$;

(ii) $\Delta_{\xi_{0}}^{\prime \prime}\left(\mathfrak{N}_{w}^{\prime}\right)^{\prime} \Delta_{\xi_{0}}^{\prime \prime}{ }^{-i t}=\left(\mathfrak{N}_{w}^{\prime}\right)^{\prime}, \forall t \in \mathbb{R}$.

Then $\mathcal{E}_{\mathfrak{N}}$ is a conditional expectation of $\left(\mathfrak{M}, \xi_{0}\right)$ w.r.t. $\mathfrak{N}$.

By Lemma 3.1 and Theorem 2.7 we deduce the following. 
Theorem 3.3. Let $\left(\mathfrak{M}, \xi_{0}\right)$ be as in Theorem 3.2 and $\mathfrak{N}$ an $O^{*}$-subalgebra of $\mathfrak{M}$. Suppose $\mathfrak{N}$ is a $G W^{*}$-algebra on $\mathcal{D}$ satisfying:

(i) $t_{\mathfrak{N}}$ is defined by a sequence $\left\{T_{n}\right\}$ of essentially self-adjoint operators in $\mathfrak{N}$ whose spectral projections leave the domain $\mathcal{D}$ invariant;

(ii) $\Delta_{\xi_{0}}^{\prime \prime}\left(\mathfrak{N}_{w}^{\prime}\right)^{\prime} \Delta_{\xi_{0}}^{\prime \prime}-i t=\left(\mathfrak{N}_{w}^{\prime}\right)^{\prime}, \forall t \in \mathbb{R}$.

Then $\mathcal{E}_{\mathfrak{N}}$ is a conditional expectation of $\left(\mathfrak{M}, \xi_{0}\right)$ w.r.t. $\mathfrak{N}$.

Proof. By Lemma 2.6. $\mathfrak{N} \xi_{0}$ and $\left\langle\mathfrak{N}_{w}^{\prime} P_{\mathfrak{N}} \mathcal{D}\right\rangle$ are essentially self-adjoint for $\mathfrak{N}$; by Theorem 3.2 it follows that $\mathcal{E}_{\mathfrak{N}}$ is a conditional expectation of $\left(\mathfrak{M}, \xi_{0}\right)$ w.r.t. $\mathfrak{N}$.

Corollary 3.4. Let $\left(\mathfrak{M}, \xi_{0}\right)$ be as in Theorem 3.2 and $\mathfrak{N}$ an $O^{*}$-subalgebra of $\mathfrak{M}$. Suppose $\mathfrak{N}$ is a $G W^{*}$-algebra on $\mathcal{D}^{\infty}(\bar{T})$, where $T$ is an essentially self-adjoint operator in $\mathfrak{N}$, and $\Delta_{\xi_{0}}^{\prime \prime}\left(\mathfrak{N}_{w}^{\prime}\right)^{\prime} \Delta_{\xi_{0}}^{\prime \prime}{ }^{-i t}=\left(\mathfrak{N}_{w}^{\prime}\right)^{\prime}, \forall t \in \mathbb{R}$. Then, $\mathcal{E}_{\mathfrak{N}}$ is a conditional expectation of $\left(\mathfrak{M}, \xi_{0}\right)$ w.r.t. $\mathfrak{N}$.

Corollary 3.5. Let $\left(\mathfrak{M}, \xi_{0}\right)$ be as in Theorem 3.2 and $\mathfrak{N}$ an $O^{*}$-subalgebra of $\mathfrak{M}$. Suppose $\mathfrak{N}$ is an $E W^{*}$-algebra on a Fréchet domain $\mathcal{D}$ satisfying $\Delta_{\xi_{0}}^{\prime \prime}{ }^{i t}\left(\mathfrak{N}_{w}^{\prime}\right)^{\prime} \Delta_{\xi_{0}}^{\prime \prime}{ }^{-i t}$ $=\left(\mathfrak{N}_{w}^{\prime}\right)^{\prime}, \forall t \in \mathbb{R}$. Then $\mathcal{E}_{\mathfrak{N}}$ can be extended to a conditional expectation $\mathcal{E}_{\mathfrak{N}_{\text {wc }}^{\prime \prime}}$ of $\left(\mathfrak{M}_{\mathrm{wc}}^{\prime \prime}, \xi_{0}\right)$ w.r.t. $\mathfrak{N}_{\mathrm{wc}}^{\prime \prime} \cdot$

\section{ACKNOWLEDGEMENT}

The second author acknowledges the hospitality of the Dipartimento di Matematica ed Applicazioni, Università di Palermo, where this work was performed.

\section{REFERENCES}

[1] J. Dixmier, Von Neumann Algebras, North-Holland, Amsterdam, 1981. MR641217 (83a:46004)

[2] A. Inoue, On a class of unbounded operator algebras, Pacific J. Math. 65, 11-95 (1976). MR 0512382(58:23641a)

[3] A. Inoue, H. Ogi, M. Takakura, Conditional expectations for unbounded operator algebras, Int. J. Math. Math. Sci. 2007, Art. ID 80152, 22 pp. MR2320785 (2008h:47159)

[4] A. Inoue, H. Ogi, M. Takakura, Conditional Expectations for $O^{*}$-algebras, Contemporary Math., 427, Amer. Math. Soc., Providence, RI, 225-234 (2007). MR2326358 (2008g:47143)

[5] R.T. Powers, Self-adjoint algebras of unbounded operators, Commun. Math. Phys. 21, 85-124 (1971). MR0283580(44:811)

[6] K. Schmüdgen, Unbounded Operator Algebras and Representation Theory, BirkhäuserVerlag, Basel, 1990. MR1056697 (91f:47062)

[7] M. Takesaki, Conditional expectations in a von Neumann algebra, J. Funct. Anal. 9, 306-327 (1972). MR0303307(46:2445)

Dipartimento di Metodi e Modelli Matematici, Facoltà d'Ingegneria, Università di Palermo, I-90128 Palermo, Italy

E-mail address: bagarell@unipa.it

Department of Applied Mathematics, Fukuoka University, Fukuoka 814-0180, Japan

E-mail address: a-inoue@fukuoka-u.ac.jp

Dipartimento di Matematica ed Applicazioni, Università di Palermo, I-90123 Palermo, ITALY

E-mail address: trapani@unipa.it 